\title{
Geometry of quantum evolution in a nonequilibrium environment
}

\author{
Xiangji Cai, ${ }^{1,}{ }^{*}$ Ruixuan Meng, ${ }^{1}$ Yanhui Zhang, ${ }^{2, \dagger}$ and Lifei Wang ${ }^{3}$ \\ ${ }^{1}$ School of Science, Shandong Jianzhu University, Jinan 250101, China \\ ${ }^{2}$ School of Physics and Electronics, Shandong Normal University, Jinan 250014, China \\ ${ }^{3}$ School of Science, Shandong Jiaotong University, Jinan 250357, China
}

\begin{abstract}
We theoretically study the geometric effect of quantum dynamical evolution in the presence of a nonequilibrium noisy environment. We derive the expression of the time dependent geometric phase in terms of the dynamical evolution and the overlap between the time evolved state and initial state. It is shown that the frequency shift induced by the environmental nonequilibrium feature plays a crucial role in the geometric phase and evolution path of the quantum dynamics. The nonequilibrium feature of the environment makes the length of evolution path becomes longer and reduces the dynamical decoherence and non-Markovian behavior in the quantum dynamics.
\end{abstract}

PACS numbers: 03.65.Yz, 05.40.-a, 02.50.-r

\section{INTRODUCTION}

The global phase related to the dynamical evolution of a quantum system contains a gauge-invariant component, namely, the geometric phase which depends only on the geometry of the path traversed by the system during the quantum evolution [1-5]. Due to the fact that a quantum system unavoidably interacts with its environment and undergoes decoherence, much extensive attention has been paid to theoretical investigations on the geometric phase in open quantum systems under nonunitary dynamics [6-26]. The geometric phase associated with quantum evolution has been observed and measured in a variety of experiments [27-36], and it has demonstrated that its geometric feature has potential applications in studying quantum phase transition and realizing geometric quantum computation [11, 37-40]. The investigation on the geometry in the dynamical evolution of an open quantum system is crucial for further understanding the origins of decoherence, quantum-classical transition and so on.

With the development of experimental techniques to control and manipulate quantum systems at different time scales and energy ranges, the study of nonMarkovian behavior in the dynamical evolution of open quantum systems has increasingly evolved into an attractive research field [41-52]. Meanwhile, the nonMarkovian effect of the dynamics in open quantum systems on the geometric phase has been well studied in equilibrium environments with both Markov and stationary statistical properties [53-56]. As a matter of fact, there are many significant situations where a nonequilibrium environment has an essential influence on the dynamical evolution of a quantum system. For example, in transient and ultrafast processes in physical or biological systems, some dynamical behavior occurs on sufficiently short time scales, and there may be no chance for the en-

\footnotetext{
*xiangjicai@foxmail.com

$\dagger$ yhzhang@sdnu.edu.cn
}

vironmental initial nonequilibrium states induced by the coupling between the system and environment to reach equilibrium rapidly [57-60].

The environment with nonstationary statistics has been taken into extensive consideration, corresponding physically to impulsively environmental excited phonons out of thermal equilibrium states initially. It has drawn much attention in the study of dynamical decoherence, geometric phases and quantum speed limits of open quantum systems in nonequilibrium environments and quantum measurements detected by a voltage-biased quantum point contact (QPC) or a single-electron transistor [6170]. Given that a quantum system may interact with a composite or structured environment, where the coupling between the sub-environments plays an essential role in the dynamical evolution of the quantum system, not only the system dynamics but the statistical properties of the environment display the non-Markovian feature, namely, the memory effect of the environmental noise [71-73]. It has been shown that the nonequilibrium feature of the environment gives rise to a Lamb-type renormalization of the intrinsic energy levels which contributes additionally to the unitary dynamical evolution of the quantum system and that the environment non-Markovian feature may not result in non-Markovian behavior in the dynamics of the quantum system $[65,68]$.

In this paper, we theoretically study the geometric effect of evolution of a two-level quantum system coupled to a nonequilibrium noisy environment. Based on the quantum master equation and geometric phase defined for nonunitary evolution, we derive the time-dependent geometric phase composed of the contributions from the dynamical evolution and the overlap between the time evolved state and initial state. We discuss how the environmental nonequilibrium feature influences the geometry of quantum evolution and explore the mechanism for the geometric effect of quantum dynamical evolution in a nonequilibrium noisy environment. It is shown that the renormalization of the intrinsic energy of the system, namely, the frequency shift induced by the nonequilibrium feature of the environment has a significant impact on both the geometric phase and the evolution path of 
the dynamics.

\section{THEORETICAL FRAMEWORK}

We consider a two-level quantum system interacting with a nonequilibrium noisy environment. The environmental effect leads to the intrinsic energy of the quantum system driven linearly by a nonstationary and nonMarkovian stochastic noise process. In an equilibrium environment, when the interaction Hamitonian is commutative with the intrinsic Hamiltonian of the system, the pure decoherence process does not cause the energy renormalization, whereas in a nonequilibrium environment, it gives rise to the renormalization of the intrinsic energy levels due to the nonstationary statistical properties of the environmental noise [65, 68].

For the pure decoherence in a nonequilibrium environment, the uncontrolled environmental degrees of freedom give rise to the stochastic fluctuations in the Hamiltonian of the quantum system as $[74,75]$

$$
H(t)=H_{0}+H_{\xi}(t)=\frac{\hbar}{2} \omega_{0} \sigma_{z}+\frac{\hbar}{2} \xi(t) \sigma_{z},
$$

where $H_{0}$ is the intrinsic Hamiltonian of the quantum system, $H_{\xi}(t)$ is the stochastic fluctuating term caused by the interaction with the environment, $\sigma_{z}$ is the Pauli matrix, $\omega_{0}$ denotes the intrinsic frequency difference between the excited state $|e\rangle$ and ground state $|g\rangle$, and $\xi(t)$ represents the environmental noise exhibiting both nonstationary and non-Markovian features. The dynamical evolution for the total density matrix yields the Liouville equation

$$
\frac{\partial}{\partial t} \rho(t ; \xi(t))=-\frac{i}{\hbar}[H(t), \rho(t ; \xi(t))] .
$$

The reduced density matrix of the quantum system can be derived by taking an ensemble average over the environmental noise as $\rho(t)=\langle\rho(t ; \xi(t))\rangle$. In a nonequilibrium environment, the dynamical evolution for the reduced density matrix of the quantum system is governed by a time-local master equation as $[67,68]$

$$
\frac{d}{d t} \rho(t)=-\frac{i}{2}\left[\omega_{0}-s(t)\right]\left[\sigma_{z}, \rho(t)\right]+\frac{1}{2} \gamma(t)\left[\sigma_{z} \rho(t) \sigma_{z}-\rho(t)\right] .
$$

Due to the nonstationary statistical properties of the environmental noise, the decoherence factor $F(t)=$ $|F(t)| e^{i \phi(t)}$ is a complex time dependent function with the modulus $|F(t)|$ and the argument $\phi(t)$ and it is employed to quantify the coherence evolution of the system initially prepared in the superposition state in the basis $\{|e\rangle,|g\rangle\}$. The quantum evolution of the reduced density matrix is closely associated with the time dependent frequency shift $s(t)$ and decoherence rate $\gamma(t)$ which are defined, respectively, as

$$
\begin{aligned}
& s(t)=-\operatorname{Im}\left[\frac{d F(t) / d t}{F(t)}\right]=-\frac{d}{d t} \phi(t), \\
& \gamma(t)=-\operatorname{Re}\left[\frac{d F(t) / d t}{F(t)}\right]=-\frac{1}{|F(t)|} \frac{d}{d t}|F(t)|,
\end{aligned}
$$

where the frequency shift $s(t)$ is used to identify the decoherence processes in equilibrium and nonequilibrium environments and the decoherence rate $\gamma(t)$ is related closely to the exchange of information between the system and environment, namely, non-Markovian behavior in the system dynamics.

To quantify the non-Markovian effect in the dynamical evolution, numerous measures for non-Markovianity in open quantum systems have been proposed based on different quantification of the distinguishability between quantum states, different divisibility properties of the quantum dynamical map and some other quantities related to the concepts in quantum information which exhibit a monotonic or an oscillating behavior in time [4245]. A most widespread measure for quantification of non-Markovianity in the dynamics of open quantum systems is based on the general notion of distinguishability of quantum states: when the distinguishability decreases, the information flows from the system into the environment, while an increase of the trace distance signifies a flow of information from the environment back into the system. Based on this measure, the non-Markovianity in the dynamical evolution of the quantum system can be generally defined by [42]

$$
\mathcal{N}=\max _{\rho_{1,2}(0)} \int_{\sigma>0} \sigma\left(t, \rho_{1,2}(0)\right) d t .
$$

where $\sigma\left(t, \rho_{1,2}(0)\right)=d D\left(\rho_{1}(t), \rho_{2}(t)\right) / d t$ denotes the rate of change of the trace distance

$$
D\left(\rho_{1}, \rho_{2}\right)=\frac{1}{2} \operatorname{tr}\left|\rho_{1}-\rho_{2}\right|,
$$

with $|A|=\sqrt{A^{\dagger} A}$ being the modulus of an operator $A$ and the bound $0 \leq D \leq 1$. It is worth pointing out that it is more convenient to employ an insensitive measure constructed in Ref. [52] to quantify the degree of non-Markovian behavior in the dynamics arising from the possible overestimate of fluctuations in the trace distance, such as in the case driven by an external field. The maximum of the trace distance difference in Eq. (5) can be obtained by taking optimization over all pairs of initial states, and thus the time-dependent non-Markovianity can be written as [47]

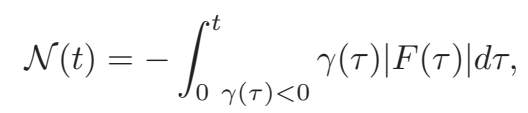

where the optimal pairs of initial states are chosen as $\rho_{1,2}(0)=\frac{1}{2}(|e\rangle \pm|g\rangle)(\langle e| \pm\langle g|)$. 
We consider the case that the environmental noise is subject to a nonstationary non-Markovian random telegraph process. The amplitude of the noise process jumps randomly with the switching rate $\lambda$ between the values $\pm \nu$, and its nonstationary and non-Markovian features are characterized by the nonequilibrium parameter $a$ and an exponential form of memory kernel $K\left(t-t^{\prime}\right)=$ $\kappa e^{-\kappa\left(t-t^{\prime}\right)}$ with the decay rate $\kappa$, respectively. The environment is in equilibrium for the nonequilibrium parameter $a=0$ when the environmental noise exhibits the stationary feature, and the environment is memoryless when the environmental noise is Markovian for the decay rate $\kappa \rightarrow \infty[65,68]$. In the presence of nonstationary non-Markovian random telegraph noise, the decoherence factor for the quantum system can be exactly written in the analytical expression [65]

$$
\begin{aligned}
& F(t)=\mathscr{L}^{-1}[\mathcal{F}(p)] \\
& \mathcal{F}(p)=\frac{p^{2}+\kappa p+2 \kappa \lambda+i a \nu(p+\kappa)}{p^{3}+\kappa p^{2}+\left(2 \kappa \lambda+\nu^{2}\right) p+\kappa \nu^{2}},
\end{aligned}
$$

where $\mathscr{L}^{-1}$ denotes the inverse Laplace transform, and the initial conditions of the decoherence factor are given by $F(0)=1$ and $\phi(0)=0$. The nonequilibrium feature of the environment only influences the imaginary component of the decoherence factor on account of its effect on the renormalization of the intrinsic energy levels of the quantum system. It is worth mentioning that the decoherence factor $F(t)$ is a real time dependent function and there is no frequency shift $s(t)=0$ for the equilibrium case.

The time dependent geometric phase for the quantum system under nonunitary dynamical evolution has been derived as [9]

$$
\begin{aligned}
\Phi_{g}(t)= & \arg \left\{\sum_{k} \sqrt{\epsilon_{k}(0) \epsilon_{k}(t)}\left\langle\Psi_{k}(0) \mid \Psi_{k}(t)\right\rangle\right. \\
& \left.\times \exp \left[-\int_{0}^{t}\left\langle\Psi_{k}(\tau)\left|\frac{\partial}{\partial \tau}\right| \Psi_{k}(\tau)\right\rangle d \tau\right]\right\},
\end{aligned}
$$

where $\epsilon_{k}(t)$ and $\left|\Psi_{k}(t)\right\rangle$ are the $k$ th time-dependent eigenvalues and eigenvectors of the reduced density matrix $\rho(t)$, respectively. Due to the environmental effect, the evolution of the quantum system is no longer cyclic and the system evolves along a quasicyclic path depending on the evolution time.

We express the state of the quantum system in terms of the Bloch vector as

$$
\rho(t)=\frac{1}{2}\left[I_{2}+\vec{r}(t) \cdot \vec{\sigma}\right],|\vec{r}(t)| \leq 1,
$$

where $I_{2}$ is the $2 \times 2$ identity matrix, $\vec{\sigma}=\left(\sigma_{x}, \sigma_{y}, \sigma_{z}\right)$ is the vector of Pauli matrices and $\vec{r}(t)=\left(r_{x}(t), r_{y}(t), r_{z}(t)\right)$ denotes a real vector with

$$
\begin{aligned}
& r_{x}(t)=\operatorname{tr}\left[\sigma_{x} \rho(t)\right]=\rho_{e g}(t)+\rho_{g e}(t), \\
& r_{y}(t)=\operatorname{tr}\left[\sigma_{y} \rho(t)\right]=i\left[\rho_{e g}(t)-\rho_{g e}(t)\right], \\
& r_{z}(t)=\operatorname{tr}\left[\sigma_{z} \rho(t)\right]=\rho_{e e}(t)-\rho_{g g}(t) .
\end{aligned}
$$

The state $\rho(t)$ is pure if and only if $|\vec{r}(t)|=1$, otherwise, mixed. Based on Eq. (3), the components of the Bloch vector $\vec{r}(t)$ satisfy the evolution

$$
\begin{aligned}
& \frac{d}{d t} r_{x}(t)=-\gamma(t) r_{x}(t)-\left[\omega_{0}-s(t)\right] r_{y}(t), \\
& \frac{d}{d t} r_{y}(t)=\left[\omega_{0}-s(t)\right] r_{x}(t)-\gamma(t) r_{y}(t), \\
& \frac{d}{d t} r_{z}(t)=0 .
\end{aligned}
$$

Consequently, the time-dependent eigenvalues of the reduced density matrix in terms of the Bloch vector can be expressed as

$$
\epsilon_{ \pm}(t)=\frac{1}{2}[1 \pm|\vec{r}(t)|],
$$

and the corresponding instantaneous eigenvectors can be written as

$$
\left|\Psi_{ \pm}(t)\right\rangle=C_{ \pm e}(t)|e\rangle+C_{ \pm g}(t)|g\rangle .
$$

with the expressions of the time dependent complex coefficients

$$
\begin{aligned}
& C_{ \pm e}(t)=\frac{r_{x}(t)-i r_{y}(t)}{\sqrt{\left[2 \epsilon_{ \pm}(t)-1-r_{z}(t)\right]^{2}+r_{x}^{2}(t)+r_{y}^{2}(t)}} \\
& C_{ \pm g}(t)=\frac{2 \epsilon_{ \pm}(t)-1-r_{z}(t)}{\sqrt{\left[2 \epsilon_{ \pm}(t)-1-r_{z}(t)\right]^{2}+r_{x}^{2}(t)+r_{y}^{2}(t)}} .
\end{aligned}
$$

If the initial state is pure, e.g., $|\vec{r}(0)|=1$, the smaller eigenvalue $\epsilon_{-}(t)$ makes no contribution to the geometric phase defined in Eq. (9) since $\epsilon_{-}(0)=0$ at time $t=0$. For the general case that the system is prepared in an arbitrary initial state, we can, based on Eq. (9), rewrite the geometric phase as

$$
\Phi_{g}(t)=\arg \left[r_{+}(t) e^{i \varphi_{+}(t)} e^{i \psi_{+}(t)}+r_{-}(t) e^{i \varphi_{-}(t)} e^{i \psi_{-}(t)}\right]
$$

where we have used the definitions

$$
\begin{aligned}
r_{ \pm}(t) & =\left|\sqrt{\epsilon_{ \pm}(0) \epsilon_{ \pm}(t)}\left\langle\Psi_{ \pm}(0) \mid \Psi_{ \pm}(t)\right\rangle\right|, \\
\varphi_{ \pm}(t) & =\arg \left\langle\Psi_{ \pm}(0) \mid \Psi_{ \pm}(t)\right\rangle, \\
\psi_{ \pm}(t) & =i \int_{0}^{t}\left\langle\Psi_{ \pm}(\tau)\left|\frac{\partial}{\partial \tau}\right| \Psi_{ \pm}(\tau)\right\rangle d \tau \\
& =\int_{0}^{t}\left[\omega_{0}+\frac{d}{d \tau} \phi(\tau)\right]\left|C_{ \pm e}(\tau)\right|^{2} d \tau .
\end{aligned}
$$

It is obvious that the geometric phase for the system evolving from an arbitrary initial state is defined as a sum over the phase factors with the weights related closely to the time-dependent eigenvalues and eigenvectors of the reduced density matrix.

For simplicity, we assume that the system is initially prepared in the pure eigenstate

$$
|\Psi(0)\rangle=\cos \frac{\theta}{2}|e\rangle+\sin \frac{\theta}{2}|g\rangle,
$$


with the initial values of the components of the Bloch vector $r_{x}(0)=\sin \theta, r_{y}(0)=0$ and $r_{z}(0)=\cos \theta$. After time $t$, the state of the quantum system evolves to

$$
|\Psi(t)\rangle=e^{-i \omega_{0} t-i \phi(t)} \cos \theta_{+}(t)|e\rangle+\sin \theta_{+}(t)|g\rangle,
$$

where the time dependent real coefficients satisfy

$$
\begin{aligned}
& \cos \theta_{+}(t)=\frac{\sin \theta|F(t)|}{\sqrt{\left[2 \epsilon_{+}(t)-1-\cos \theta\right]^{2}+\sin ^{2} \theta|F(t)|^{2}}}, \\
& \sin \theta_{+}(t)=\frac{2 \epsilon_{+}(t)-1-\cos \theta}{\sqrt{\left[2 \epsilon_{+}(t)-1-\cos \theta\right]^{2}+\sin ^{2} \theta|F(t)|^{2}}},
\end{aligned}
$$

with the larger eigenvalue $\epsilon_{+}(t)$ of the reduced density matrix which only makes a contribution to the geometric phase

$$
\epsilon_{+}(t)=\frac{1}{2}\left[1+\sqrt{\cos ^{2} \theta+\sin ^{2} \theta|F(t)|^{2}}\right] .
$$

Thus, the time dependent geometric phase in Eq. (16) can be expressed as

$$
\Phi_{g}(t)=\Phi_{P}(t)+\Phi_{e}(t),
$$

which contains the contribution arising from the overlap between the time evolved state $|\Psi(t)\rangle$ and initial state $|\Psi(0)\rangle$, namely, the Pancharatnam relative phase $[1,9]$, which can be written by

$$
\begin{aligned}
\Phi_{P}(t) & =\arg \langle\Psi(0) \mid \Psi(t)\rangle \\
& =-\arctan \frac{\sin \left[\omega_{0} t+\phi(t)\right]}{\cos \left[\omega_{0} t+\phi(t)\right]+\tan \frac{\theta}{2} \tan \theta_{+}(t)},
\end{aligned}
$$

and the contribution resulting from the geometric effect of the dynamical evolution, namely, the effective geometric phase, which can be expressed as

$$
\begin{aligned}
\Phi_{e}(t) & =i \int_{0}^{t}\left\langle\Psi(\tau)\left|\frac{\partial}{\partial \tau}\right| \Psi(\tau)\right\rangle d \tau \\
& =\int_{0}^{t}\left[\omega_{0}-s(\tau)\right] \cos ^{2} \theta_{+}(\tau) d \tau .
\end{aligned}
$$

The reason why the phase in Eq. (24) is called the effective geometric phase is that $\Phi_{e}(t)$ is closely associated with the dynamical evolution and that for a given evolution time, the Pancharatnam relative phase $\Phi_{P}(t)$ in Eq. (23) maybe disappear and makes no contribution to the dynamical evolution. For the case that the system evolves along a quasicyclic path with the evolution time $t=2 \pi / \omega_{0}$, the expression of the geometric phase in Eq. (22) is consistent with that obtained in Refs. [18, 19]. Obviously, in contrast to that in an equilibrium environment, the renormalization of the intrinsic energy of the system induced by the environmental nonequilibrium feature, namely, the frequency shift $s(t)$ gives an additional contribution to the geometric phase.
It is worth mentioning the situation when the environment is in equilibrium $(a=0)$ and the evolution of the system is quasicyclic with time $t=2 \pi / \omega_{0}$. In this case, there is no frequency shift and the decoherence factor $F(t)$ is real with zero argument. As a consequence, the Pancharatnam relative phase in Eq. (23) is zero and the geometric phase in Eq. (22) only arises from the effective geometric phase of the dynamical evolution which can be reduced to

$$
\Phi_{g}(t)=\Phi_{e}(t)=\omega_{0} \int_{0}^{t} \cos ^{2} \theta_{+}(\tau) d \tau .
$$

This expression returns to the well-known results obtained in Refs. [14, 16, 54].

The effective geometric phase in Eq. (24) can be expressed, by making the correction, as [16]

$$
\Phi_{e}(t)=\Phi_{e}^{U}(t)+\delta \Phi_{e}(t),
$$

where $\Phi_{e}^{U}(t)=\omega_{0} t \cos ^{2}(\theta / 2)$ denotes the unitary effective geometric phase with no influence from the environment, and $\delta \Phi_{e}(t)$ is the correction to the effective geometric phase made between the cases under nonunitary and unitary dynamical evolution. For the case under unitary dynamics and evolution time $t=2 \pi / \omega_{0}$, the effective geometric phase can be reduced to $\Phi_{e}^{U}=\pi(1+\cos \theta)$. Combining Eqs. (24) with (26), it indicates that the frequency shift $s(t)$ induced by the environmental nonequilibrium feature also has a significant impact on the correction to the effective geometric phase.

\section{RESULTS AND DISCUSSION}

In this section, we show the results of the geometric effect of the dynamical evolution in Markovian and non-Markovian regions induced by the nonequilibrium environment. We mainly focus on the influence of the environmental nonequilibrium feature on the geometric effect of the quantum dynamics. For simplicity, we set $\omega_{0}=0$ and use the case for the environment in equilibrium $(a=0)$ as a reference. In this case, the effective geometric phase can indirectly reflect the correction to the unitary geometric phase.

Figure 1 shows the time evolution of the effective geometric phase $\Phi_{e}(t)$ for different environmental nonequilibrium parameter $a$ in Markovian and non-Markovian dynamics regions, respectively. When the environment is out of equilibrium, in both dynamics regions, $\Phi_{e}(t)$ shows symmetrical behavior on opposite sides of the equilibrium case for positive and negative $a$. As time goes by, in Markovian dynamics region as shown in Fig. 1(a), $\Phi_{e}(t)$ gradually tends to a stable value whereas it increases monotonically in non-Markovian dynamics region as shown in Fig. 1(b). Furthermore, as the environment departs from equilibrium for a given evolution time, in Markovian dynamics region, $\Phi_{e}(t)$ deviates from the equilibrium case; on the contrary, the behavior of deviation is not obvious in non-Markovian dynamics region. 

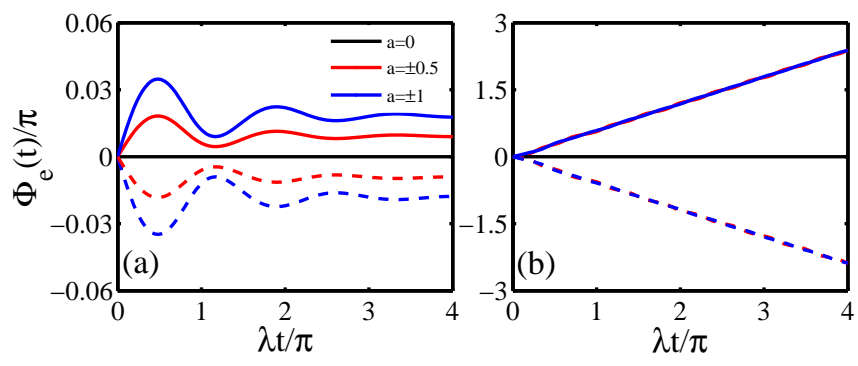

FIG. 1. (Color online) Effective geometric phase $\Phi_{e}(t)$ as a function of evolution time $t$ for different values of $a$ with $\theta=\pi / 2$ in (a) Markovian dynamics region with $\nu=0.5 \lambda$ and $\kappa=\lambda$ and (b) non-Markovian dynamics region with $\nu=2 \lambda$ and $\kappa=\lambda$ (the solid and dashed lines are plotted for $a>0$ and $a<0$, respectively).

This suggests that the environmental nonequilibrium feature gives different additional contributions to the geometric effect of the dynamical evolution in the two dynamics regions and that the correction to the geometry of quantum evolution is mainly ruled by the nonequilibrium feature of the environment in Markovian dynamics region. Furthermore, the effective geometric phase $\Phi_{e}(t)$ in non-Markovian dynamics region is much larger than that in Markovian dynamics region, which indicates that the non-Markovian behavior in the dynamics makes large correction to the geometric effect of the dynamical evolution. This shows very good agreement with the conclusions obtained in Ref. [64].
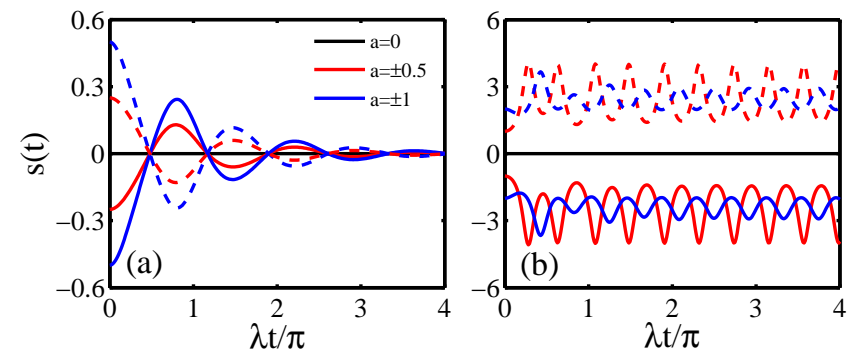

FIG. 2. (Color online) Time evolution of the frequency shift $s(t)$ for different values of $a$ with $\theta=\pi / 2$ and $\kappa=\lambda$ in (a) Markovian dynamics region with $\nu=0.5 \lambda$ and (b) nonMarkovian dynamics region with $\nu=2 \lambda$ (the solid and dashed lines are also for positive and negative $a$, respectively).

To study the reason of difference in the effective geometric phase between the equilibrium and nonequilibrium cases, we show the frequency shift $s(t)$ for different values of $a$ in Markovian and non-Markovian dynamics regions in Fig. 2(a) and (b), respectively. In both dynamics regions, $s(t)$ shows the symmetry for a taking positive and negative values. When the environment is in nonequilibrium, in Markovian dynamics region, $s(t)$ decays with oscillatory behavior and discrete zeros and it asymptotically approaches zero as time goes on. How- ever, $s(t)$ oscillates periodically in time with nonzero midline in non-Markovian dynamics region. Furthermore, as the environment deviates from equilibrium for a given evolution time, $s(t)$ increases in Markovian dynamics region whereas it hardly changes in non-Markovian dynamics region. The behavior in time-dependent frequency shift $s(t)$ is closely associated with the effective geometric phase as shown in Fig. 1. It further indicates that the environmental nonequilibrium feature which gives rise to the renormalization of the intrinsic energy of the quantum system plays an important role in the geometric effect of the dynamical evolution.
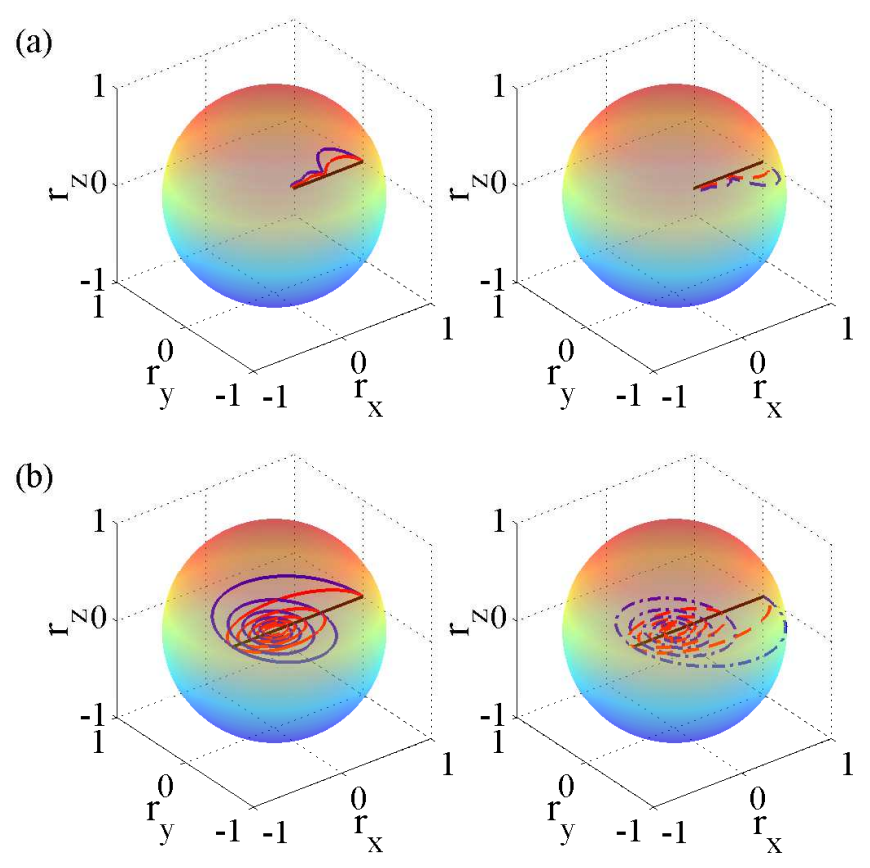

FIG. 3. (Color online) Geometry of the dynamical evolution of the quantum system in Bloch sphere representation (a) Markovian dynamics region with $\nu=0.5 \lambda$ and $\kappa=\lambda$ and (b) non-Markovian dynamics region with $\nu=2 \lambda$ and $\kappa=\lambda$. The left and right columns are plotted for $a>0$ and $a<0$, respectively. Blue line for $a= \pm 1$, red line for $a= \pm 0.5$ and black line for $a=0$.

In order to study how the environmental nonequilibrium feature influences the path of the dynamical evolution, we show the time evolution of the reduced density matrix of the system for different values of $a$ in the Bloch sphere representation in Fig. 3. This helps us to understand better the combined effects of the unitary and nonunitary parts of dynamical evolution which are closely associated with the energy renormalization and dynamical decoherence. The radius $|\vec{r}(t)|$ of the Bloch vector denotes the absolute value of the decoherence factor: the normal and tangential slopes of the radius are related to the decoherence rate and frequency shift, respectively, and the rate of change of the radius is associated with non-Markovian behavior in the system dynamics. Obviously, when the environment is out of equilibrium, the 
geometric evolution path displays antisymmetrical behavior in the Bloch sphere for positive and negative $a$ in both dynamics regions. In Markovian dynamics region as shown in Fig. 3(a), $|\vec{r}(t)|$ decays monotonically whereas as shown in Fig. 3(b) in non-Markovian dynamics region, it decays with periodical oscillations, namely coherence revivals induced by environmental backaction. Furthermore, in non-Markovian dynamics region, the oscillatory behavior in $|\vec{r}(t)|$ gets reduced as the environment departs from equilibrium, which reflects that the environmental nonequilibrium feature can suppress nonMarkovian behavior in the system dynamics. Moreover, as the environment deviates from equilibrium in both dynamics regions, the length of evolution path becomes longer which suggests that the environmental nonequilibrium feature reduces the dynamical decoherence of the quantum system.

\section{CONCLUSIONS}

In this paper, we have studied the geometry of dynamical evolution of a two-level quantum system coupled to a nonequilibrium noisy environment. Due to the nonstationary statistical properties of the environmental noise, the decoherence factor is a complex time-dependent function and the imaginary part of the decoherence factor gives an additional contribution to the unitary evolution of the system dynamics. Based on the quantum master equation in a nonequilibrium environment, we derived the time evolution of the geometric phase closely associated with the renormalization of the intrinsic energy of the system, namely, the frequency shift. We have demon- strated that the environmental nonequilibrium feature plays a crucial role in both the geometric phase and evolution path of the quantum dynamics. It was shown that the nonequilibrium feature of the environment makes the length of evolution path becomes longer and reduces the dynamical decoherence of the quantum system compared with the equilibrium case. This result is significant to quantum information processing based on the geometry of dynamical evolution of open quantum systems.

The investigation on the geometric effect of dynamical evolution in a nonequilibrium environment helps us understand better the non-Markovian decoherence dynamics of open quantum systems. Within some theoretical and experimental frameworks, the phase information of quantum evolution can be measured by the interferometric measurement via nuclear magnetic resonance (NMR) or by the current measurement via a QPC device $[18,32,33,69,70]$. In principle, the observation of the environmental nonequilibrium feature on the geometry of dynamical evolution would be expected to be realized experimentally by using a NMR interferometry or a QPC detector based on the theoretical frameworks demonstrated in Refs. [18] and [70], respectively.

\section{ACKNOWLEDGMENTS}

This work was supported by the Natural Science Foundation of Shandong Province under Grant Nos. ZR2014AM030 and ZR2016AP14. X.C. and R.M. also acknowledge the support from the Doctoral Research Fund of Shandong Jianzhu University (Grant Nos. XNBS1852 and XNBS1860).
[1] S. Pancharatnam, Proc. Ind. Acad. Sci. A 44, 247 (1956).

[2] M. V. Berry, Proc. R. Soc. A 392, 45 (1984).

[3] A. Shapere and F. Wilczek, Geometric Phases In Physics (World Scientific, London, 1989).

[4] C. A. Mead, Rev. Mod. Phys. 64, 51 (1992).

[5] A. Bohm, A. Mostafazadeh, H. Koizumi, Q. Niu, and J. Zwanziger, The Geometric Phase in Quantum Systems (Springer, New York, 2003).

[6] Y. Aharonov and J. Anandan, Phys. Rev. Lett. 58, 1593 (1987).

[7] J. Samuel and R. Bhandari, Phys. Rev. Lett. 60, 2339 (1988).

[8] A. Carollo, I. Fuentes-Guridi, M. F. m. c. Santos, and V. Vedral, Phys. Rev. Lett. 90, 160402 (2003).

[9] D. M. Tong, E. Sjöqvist, L. C. Kwek, and C. H. Oh, Phys. Rev. Lett. 93, 080405 (2004).

[10] D. M. Tong, E. Sjöqvist, S. Filipp, L. C. Kwek, and C. H. Oh, Phys. Rev. A 71, 032106 (2005).

[11] A. C. M. Carollo and J. K. Pachos, Phys. Rev. Lett. 95, 157203 (2005).

[12] A. Carollo, G. Massimo Palma, A. Łozinski, M. F. m. c. Santos, and V. Vedral, Phys. Rev. Lett. 96, 150403 (2006).
[13] X. X. Yi, L. C. Wang, and W. Wang, Phys. Rev. A 71, 044101 (2005).

[14] X. X. Yi, D. M. Tong, L. C. Wang, L. C. Kwek, and C. H. Oh, Phys. Rev. A 73, 052103 (2006).

[15] A. Bassi and E. Ippoliti, Phys. Rev. A 73, 062104 (2006).

[16] F. C. Lombardo and P. I. Villar, Phys. Rev. A 74, 042311 (2006).

[17] X. X. Yi and W. Wang, Phys. Rev. A 75, 032103 (2007).

[18] F. M. Cucchietti, J.-F. Zhang, F. C. Lombardo, P. I. Villar, and R. Laflamme, Phys. Rev. Lett. 105, 240406 (2010).

[19] P. I. Villar and F. C. Lombardo, Phys. Rev. A 83, 052121 (2011).

[20] J. Hu and H. Yu, Phys. Rev. A 85, 032105 (2012).

[21] X.-D. Cui and Y. Zheng, Phys. Rev. A 86, 064104 (2012).

[22] M. Pechal, S. Berger, A. A. Abdumalikov, J. M. Fink, J. A. Mlynek, L. Steffen, A. Wallraff, and S. Filipp, Phys. Rev. Lett. 108, 170401 (2012).

[23] F. C. Lombardo and P. I. Villar, Phys. Rev. A 89, 012110 (2014).

[24] F. C. Lombardo and P. I. Villar, Europhys. Lett. 118, 50003 (2017).

[25] D.-W. Luo, J. Q. You, H.-Q. Lin, L.-A. Wu, and T. Yu, 
Phys. Rev. A 98, 052117 (2018).

[26] A. Carollo, B. Spagnolo, and D. Valenti, Sci. Rep. 8, 9852 (2018).

[27] R. Y. Chiao and Y.-S. Wu, Phys. Rev. Lett. 57, 933 (1986).

[28] A. Tomita and R. Y. Chiao, Phys. Rev. Lett. 57, 937 (1986).

[29] T. Bitter and D. Dubbers, Phys. Rev. Lett. 59, 251 (1987).

[30] D. Suter, K. T. Mueller, and A. Pines, Phys. Rev. Lett. 60, 1218 (1988).

[31] P. J. Leek, J. M. Fink, A. Blais, R. Bianchetti, M. Göppl, J. M. Gambetta, D. I. Schuster, L. Frunzio, R. J. Schoelkopf, and A. Wallraff, Science 318, 1889 (2007).

[32] J. Du, P. Zou, M. Shi, L. C. Kwek, J.-W. Pan, C. H. Oh, A. Ekert, D. K. L. Oi, and M. Ericsson, Phys. Rev. Lett. 91, 100403 (2003).

[33] J. Du, J. Zhu, M. Shi, X. Peng, and D. Suter, Phys. Rev. A 76, 042121 (2007).

[34] M. Möttönen, J. J. Vartiainen, and J. P. Pekola, Phys. Rev. Lett. 100, 177201 (2008).

[35] H. Chen, M. Hu, J. Chen, and J. Du, Phys. Rev. A 80, 054101 (2009).

[36] Z. Zhang, T. Wang, L. Xiang, J. Yao, J. Wu, and Y. Yin, Phys. Rev. A 95, 042345 (2017).

[37] S.-L. Zhu, Phys. Rev. Lett. 96, 077206 (2006).

[38] J. A. Jones, V. Vedral, A. Ekert, and G. Castagnoli, Nature 403, 869 (2000).

[39] P. Solinas, P. Zanardi, N. Zanghì, and F. Rossi, Phys. Rev. B 67, 121307 (2003).

[40] L. Faoro, J. Siewert, and R. Fazio, Phys. Rev. Lett. 90, 028301 (2003).

[41] H. P. Breuer and F. Petruccione, The Theory of Open Quantum Systems (Oxford University Press, New York, 2002).

[42] H. Breuer, E. Laine, and J. Piilo, Phys. Rev. Lett. 103, 210401 (2009).

[43] A. Rivas, S. F. Huelga, and M. B. Plenio, Phys. Rev. Lett. 105, 050403 (2010).

[44] W.-M. Zhang, P.-Y. Lo, H.-N. Xiong, M. W.-Y. Tu, and F. Nori, Phys. Rev. Lett. 109, 170402 (2012).

[45] D. Chruściński and S. Maniscalco, Phys. Rev. Lett. 112, 120404 (2014).

[46] A. Rivas, S. F. Huelga, and M. B. Plenio, Rep. Prog. Phys. 77, 094001 (2014).
[47] H. Breuer, E. Laine, J. Piilo, and B. Vacchini, Rev. Mod. Phys. 88, 021002 (2016).

[48] I. de Vega and D. Alonso, Rev. Mod. Phys. 89, 015001 (2017).

[49] A. Chenu, M. Beau, J. Cao, and A. del Campo, Phys. Rev. Lett. 118, 140403 (2017).

[50] L. Magazzù, A. Carollo, B. Spagnolo, and D. Valenti, J. Stat. Mech. 2016, 054016 (2016).

[51] Y.-A. Yan and J. Shao, Phys. Rev. A 97, 042126 (2018).

[52] P. M. Poggi, F. C. Lombardo, and D. A. Wisniacki, Europhys. Lett. 118, 20005 (2017).

[53] X. L. Huang and X. X. Yi, Europhys. Lett. 82, 50001 (2008).

[54] J.-J. Chen, J.-H. An, Q.-J. Tong, H.-G. Luo, and C. H. Oh, Phys. Rev. A 81, 022120 (2010).

[55] S. L. Wu, X. L. Huang, L. C. Wang, and X. X. Yi, Phys. Rev. A 82, 052111 (2010).

[56] W. Guo, J. Ma, X. Yin, W. Zhong, and X. Wang, Phys. Rev. A 90, 062133 (2014).

[57] C. C. Martens, J. Chem. Phys. 133, 241101 (2010).

[58] C. C. Martens, J. Phys. B 45, 154008 (2012).

[59] M. Schiró and A. Mitra, Phys. Rev. Lett. 112, 246401 (2014).

[60] F. Peronaci, M. Schiró, and M. Capone, Phys. Rev. Lett. 115, 257001 (2015).

[61] C. Emary, Phys. Rev. A 78, 032105 (2008).

[62] C. C. Martens, J. Chem. Phys. 139, 024109 (2013).

[63] F. C. Lombardo and P. I. Villar, Phys. Rev. A 87, 032338 (2013).

[64] F. C. Lombardo and P. I. Villar, Phys. Rev. A 91, 042111 (2015).

[65] X. Cai and Y. Zheng, Phys. Rev. A 94, 042110 (2016).

[66] C. Wang, J. Ren, and J. Cao, Phys. Rev. A 95, 023610 (2017).

[67] X. Cai and Y. Zheng, Phys. Rev. A 95, 052104 (2017).

[68] X. Cai and Y. Zheng, J. Chem. Phys. 149, 094107 (2018).

[69] L. Kang, Y. Zhang, X. Xu, and X. Tang, Phys. Rev. B 96, 235417 (2017).

[70] B. Liu, F.-Y. Zhang, J. Song, and H.-S. Song, Sci. Rep. 5, 11726 (2015).

[71] P. Hänggi and H. Thomas, Phys. Rep. 88, 207 (1982).

[72] A. Fuliński, Phys. Rev. E 50, 2668 (1994).

[73] A. Fuliński, Europhys. Lett. 118, 60002 (2017).

[74] P. W. Anderson, J. Phys. Soc. Jpn. 9, 316 (1954).

[75] R. Kubo, J. Phys. Soc. Jpn. 9, 935 (1954). 